
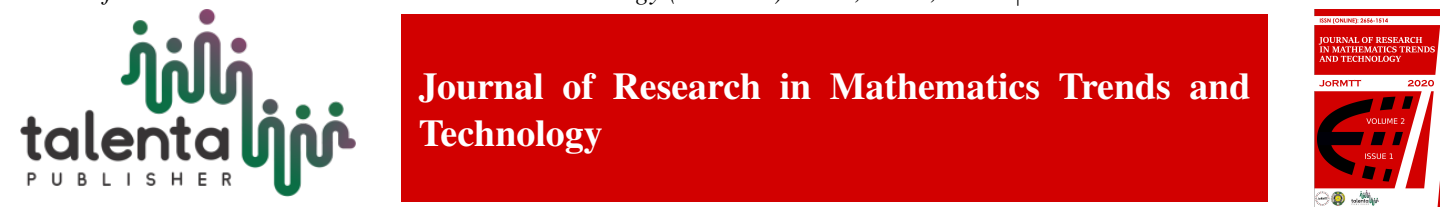

\title{
Existence of Polynomial Combinatorics Graph Solution
}

\author{
Mardiningsih $^{1^{*}}$, Saib Suwilo ${ }^{1}$ and Ihda Hasbiyati ${ }^{2}$ \\ ${ }^{1}$ Department of Mathematics, Universitas Sumatera Utara, Medan, 20155, Indonesia \\ ${ }^{2}$ Department of Mathematics, Universitas Riau, Riau, 28293, Indonesia
}

\begin{abstract}
The Polynomial Combinatorics comes from optimization problem combinatorial in form the nonlinear and integer programming. This paper present a condition such that the polynomial combinatorics has solution. Existence of optimum value will be found by restriction of decision variable and properties of feasible solution set or polyhedra.

Keywords: Optimum Value, Polyhedra, Polynomial Combinatorics

Abstrak. Kombinatorika Suku Banyak berasal dari permasalahan optimisasi kombinatorial dalam bentuk pemrograman nonlinear dan integer. Makalah ini memberikan suatu kondisi sehingga kombinatorika suku banyak memiliki solusi. Eksistensi dari nilai optimum akan diperoleh dengan membatasi variabel keputusan dan sifat-sifat dari himpunan solusi yang layak atau polihedra.
\end{abstract}

Kata Kunci: Nilai Optimum, Polihedra, Kombinatorika Suku Banyak

Received 10 December 2019 | Revised 9 January 2020 | Accepted 13 February 2020

\section{Introduction}

An optimization problem is started with a set of variables or parameters, and often include conditions or restrictions on the obtained value of the variable. Such restriction is termed the constraints of the problem. Another important component of the optimization problem is called the objective function, which depends on variables of problem. The solution of the optimization problem is the set of values of variables that satisfy the constraint, so that the objective function reaches the optimum value.

Combinatorial optimization problems can be formulated in graph form and in the form of mathematics program. A combinatorial optimization problem in a graph that has many applications and has been studied intensively is the graph coloring problem [1]. Problems of graph coloring of a graph $G=(V, E)$, is a problem of searching for the minimum number of colors that can be given at any point in the set $V$ with each point given one color, with the constraints for any edge $(i, j) \in E$, the color used for the vertex $i$ and vertex $j$ must be different, this problem is a combinatorial optimization problem. In this paper studied combinatorial optimization problems which special math program has the objective function and constraints in the form of polynomials, and hereinafter referred to as polynomial combinatorics. The general form of a polynomial of combinatorics is:

Maximize $f(X)$

*Corresponding author at: Department of Mathematics, Universitas Sumatera Utara, Medan, 20155, Indonesia

E-mail address: mardiningsih@usu.ac.id 


$$
\begin{gathered}
g_{i}(X)=0, i=1,2, \ldots, m \\
g_{i}(X), \quad i=m+1, m+2, \ldots, m \\
l \leq X \leq u
\end{gathered}
$$

with $f, g_{i} \in \mathbb{Z}[x]$ and $X=\left\{\left(a_{1}, \ldots, a_{d}\right) \mid a_{1}, \ldots, a_{d} \in \mathbb{Z}\right\}$ is the set of all polynomials with $d$ variables and with integer coefficients.

Lorea.et al (2008) showed the solution of combinatorial optimization problem in graph based on the existence of the completion of the its combinatorics polynomial. It has been proved that combinatorial problems have solution if and only if the its combinatorics polynomial have a the solution.

Based on the description above, shows that how important to be known that a combinatorial polynomial has a solution, but until now there is no guarantee that a combinatorial polynomial has solution. But to determine that the combinatorial polynomial does not have solution, there is a guarantee that is called collateral Nullstelensatz [2]. Therefore, it is necessary to study what that guarantees a combinatorial polynomial has solution.

The conditon of optimality should be given before investigating searching of optimality condition in order polynomial combinatorics (1) has a solution (resolved). needs to be studied several cases that have been investigated by previous researchers. A model (1) has a the solution (resolved) is obtained the set of decision variables (point integers) that satisfies all the constraints is called set of feasible or polyhedron $\left(P=\left\{x \in X \mid g_{i}(x) \leq 0\right\}\right)$ is not an empty set, so the objective function $f$ achieve optimum value

There are two possibilities that occur when a polynomial combinatorial problems do not have solutions, namely for example when the problem infeasible or its polyhedra is an empty set and $f$ is unlimited. A necessary condition that $f(x)$ has a relative minimum at $x=x^{*}$, is $f(x)$ should be defined on an interval $[a, b]$ for $a<x^{*}<b$ and if the derivative $f(x)$ or $\Delta f(f$ multivariable) exist at $x=x^{*}$, then $f\left(x^{*}\right)$ or $\Delta f\left(x^{*}\right)=0$. A sufficient condition for the minimum value of a local or global of $f(x)$ if $f(x)=0, f^{\prime}(x)=0$, until to $f^{(n 1)}(x)=0$ and $f^{(n)}(x)>0$ for $n$ is even, or Hess matrix at $x=x^{*}$ is positive definite.

Since $f$ is a polynomial then the maximum value of $f(x)$ if exists in its polyhedra is not singular, that there is a local maximum and there is a global maximum (optimal value), so there must be a guarantee that the maximum value local is a global maximum value, namely if the function $f: S \rightarrow$ $X$ on $S \subseteq X$, assuming a convex subset $S$ and $f$ is also convex function of the local maximum is a global maximum. At [3] have discussed the issue of feasible area or a polynomial combinatorial polyhedron, specifically with linear objective function and constraints of any polynomial with integer coefficients. It also defines the convexity problem a function at integer, as an extension of the definition of convexity on a continuous function in $R$, also examines the properties of the polyhedron obtained.

The purpose of this paper is to present a polynomial combinatorial condition that (1) has a solution. Before discussing the existence of the completion of the optimization polnomial with integer co- 
efficients and integer decision variables or discuss settlement combinatorial polynomial, it should be studied polynomial optimization problem. Polynomial optimization referred to in this article is a mathematical programming problem with the objective and constraint function is polynomials, with decision variables is real numbers.

\section{The Existence of Polynomial Optimization Solution}

The question of this equation (1) is about the existence of the optimal solution of this model. As in the linear programming problem, the solution may not exist. As well as for problems of this polynomial program, there is possibility that does not have a solution. Criteria which guarantee the existence of the optimal solution of this problem is based on the continuity of the objective function $f$ and $g$ constraint function. Before discussing the existence guarantee of optimal solution of these problems, need to be defined some of the terms used.

Definition 1. Polyhedra $P$ on or in is a set of all real numbers or integers that satisfy all constraints of an optimization problem. For equation (1), its polyhedra is

$$
P=\{x \in X \mid g(x) \leq 0\}
$$

Theorem 2. Assume $f, g$, at the model (1) is a continuous function and satisfy the following statement: 1 . The set of feasible points $g(x)$ is a finite set in 2 . The set of feasible points is not limited, but

$$
\lim _{|x| \rightarrow \infty, g(x) \leq 0} f(x)=+\infty,
$$

then the polynomial optimization problem (1) have at least one solution.

Optimization polynomial (1) has the objective function $\mathrm{f}$ and constraint $\mathrm{g}$ with polynomial form then $\mathrm{f}$ and $\mathrm{g}$ is continuous on its domain, as well as the value of the decision variable $\mathrm{x}$ is limited, so that $\mathrm{g}(\mathrm{x})$ limited, consequently the problem (1) have at least one solution, because it satisfy Theorem 1.

This problem only guarantees the existence of the settlement of the problem (1), but it is not known that the obtained solution is an optimal solution (global) or a local solution. Therefore, needed a requirement which ensures the optimal solution. In this section discussed how to detect a necessary condition of optimality that must be met at a solution point for 1 local maximum or local minimum. Because polynomial in an interval (domain) can be obtained minimum value (if exist) more than one value, then there is no guarantee that which is the global minimum value.

Henceforth in this paper, the global minimum value is called optimal value. Before knowing which ensures that a local minimum value is the optimum value, it takes the following definitions and theorems.

\subsection{Convexity of Integer}

Definition 3. A set $K \subset \mathbb{Z}^{n}$ is convex if for every pair of vector $x, y \in K$ apply,

$$
\alpha x+(1-\alpha) y \in K \quad \text { for all } \alpha \in[0,1] .
$$

Definition 4. A function $f: K \rightarrow \mathbb{Z}$ is called convex if $K$ is the set of convex and for all $x, y \in K$ 
apply;

$$
f(\alpha x+(1-\alpha) y) \geq \alpha f(x)+(1-\alpha) f(y), \quad \text { for all } \alpha \in[0,1] .
$$

Theorem 5. Suppose $x, y \in K, f: K \rightarrow \mathbb{Z}$, is a convex function and $K$ is a convex set. If $x_{0}$ is the local minimum value of $f$ in $K$ then $x_{0}$ is also a global minimum value of $f$ in $K$.

\section{Combinatorial Polynomial Problem}

In this chapter is presented a problem of existence of combinatorial polynomial solution. Solution of combinatorial polynomial is the set of values of decision variables of integers that satisfy limit of polynomial constraint function that is called feasible set (polyhedra), such that polynomial objective function reaches the optimum value.

Before discussing condition that guarantee the problem of combinatorial polynomial have solution. At first, discuss the relationship between the feasible set (polyhedra) with the optimum value of the objective function. The second section discusses the set of settlement (polyhedra) and its properties. The third discusses the existence or what the optimum value which ensures that at least one integer in the polyhedron which is the optimum value that meets the objective function.

In order to facilitate discussion polynomial combinatorial problem solving, needs to be presented general form of mathematical models which is representative. The general forms of polynomial combinatorics is:

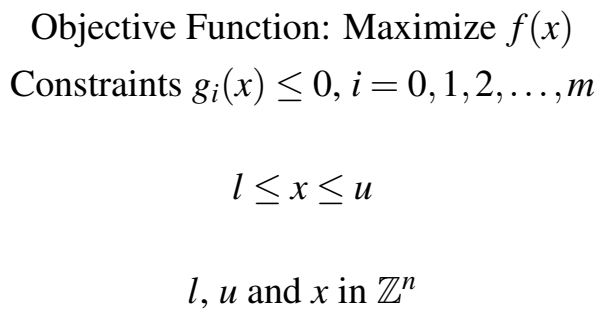

with $f, g_{i}$ in $\mathbb{Z}[x]$.

Completion of the polynomial combinatorics (2), is the value of the decision variable $x$ which satisfy the constraint $g$ such that obtained the optimum value of the objective function $f(x)$ constraints polynomial $g$ affect the optimum value of $f$. Whether or not the settlement depends on the influence of constraint functions $g$ to contour of objective function $f$.

\subsection{Feasible Set (Polyhedra)}

Polyhedra of problems (2) is the set of integers that satisfy all constraints, denoted by $S_{B}=\{x \in$ $\left.\mathbb{Z}^{n} \mid g_{i}(x) \leq 0, i \in M, l \leq x \leq u\right\}$ with $g_{i} \in \mathbb{Z}[x]$ for all $i \in M=1, \ldots, m$ and $l, u$ and $l>0$ and $u>0$. From the above explanation, to obtain optimal values of a polynomial combinatorics, need question how to determine those who are points of integers that satisfy all constraints (polyhedra). And based on polyhedra obtained, will then be used to search terms or assumption of what was given to ensure the existence of at least one integer on a polyhedron which is the optimum value (local or global). The solution of combinatorial polynomial problem (2) is the value of the decision 
variable $x \in S_{B}$ which generate optimal value $f(x)$ are presented as,

$$
\text { Minimum } f(x) \text { for } x \in S_{B}
$$

with $S_{B}=\left\{x \in \mathbb{Z}^{n} \mid g_{i}(x) \leq 0, i \in M, l \leq x \leq u\right\}$. So it will be looked for the optimum value of $f$ on the set $S_{B}$. In general, can not be known whether the problem (2) can be resolved or not, therefore it needs to be presented condition for $f$ and $S_{B}$ which ensures the problem (2) can be completed (having settlement).

The minimum value (local or global) of optimization (2) is dependent upon the set formed by the constraints of $g$, because it could be the objective function $f$ does not fulfill all the constraints $g$. Therefore, there is a requirement constraints on the $l \leq x \leq u$. For the upper limit of $u$, should be given on condition that $u \in \mathbb{Z}^{n}$ and $u>0$, for each variable is limited to integer $x$, with $x \in \cap[l, u]$, can be transformed into an non-negative integer variables other for. For example $x=x l$, with an upper limit $u=u l$. To ensure there are members in the soltion set, the optimization problem given should be defined, namely the set of settlement (polyhedra) should also be convex. Because of these problems associated with integers, it would be required the definition of a integer convex function. So, it ensures at least there exist one element in the polyhedron.

After finding the existence of a set of settlement of the problem (2), the next will be discussed the set of all integers minimum point. To examine the set of all integer points of constraint of combinatorial polynomial combinatorial optimization problem (2) required steps:

1. Collect integer points that satisfies every constraint (polyhedra) $S_{B}$ and convex hull $P_{[g]}$

$$
\begin{aligned}
S_{B} & =\left\{x \in \mathbb{Z}^{n} \mid g_{i}(x) \leq 0, i \in M, l \leq x \leq u\right\} \\
P_{[g]} & =\operatorname{conv}\left(\left\{x, g(x) \in \mathbb{Z}[x] \mid g_{i}(x) \leq 0, l \leq x \leq u\right\}\right)
\end{aligned}
$$

with $g(x)$ denotes the values $\left(g_{1}(x), \ldots, g_{m}(x)\right) \in \mathbb{Z}[x]$ and $g=\left(g_{1}, \ldots, g_{m}\right) \in \mathbb{Z}[x]^{m}$

2. Shows ties $S_{B}$ with $P_{[g]}$

3. To show the relationship $S_{B}$ with $P_{[g]}$ takes a few definitions and lemma.

Definition 6. Suppose the vector $\left(x_{1}, x_{2}, \ldots, x_{k}\right) \in \mathbb{Z}$ and $\lambda_{1}, \lambda_{2}, \ldots, \lambda_{k} \geq 0$ to $\sum_{i=1}^{k} \lambda_{i}=1$, then $x=$ $\sum_{i=1}^{k} \lambda_{i} x_{i}$ is called a convex combination of $x_{1}, x_{2}, \ldots, x_{k}$. Convex hull with the notation $\operatorname{conv}(X)$ of the set $X$ defined as the set of all convex combinations of points in $X$.

Definition 7. Projections of polyhedra $Q \subseteq \mathbb{R}^{n+m}$ on $\mathbb{R}^{n}$, at $x^{0} \in S_{B}$ is denoted by:

$$
\mathbb{Q}_{x}=\left\{x \in \mathbb{R}^{n} / \exists y \in \mathbb{R}^{m}(x, y) \in \mathbb{Q}\right\}
$$

Lemma 8. Let $g \in \mathbb{Z}[x]^{m}$, if

$$
S_{B}=\left\{x \in \mathbb{Z}^{n} \mid g_{i}(x) \leq 0, i \in M, l \leq x \leq u\right\}
$$

then

$$
S_{B} \subseteq\left(P_{[g]} \cap\left\{(x, y) \in \mathbb{R}^{n+m} / y \leq 0\right\}\right)
$$


Furthermore, to show the relationship $S_{B}=P_{[g]}$, we need the definitions of convexity of integer on subsection 2.1, which is expansion of the definition of real numbers.

Theorem 9. Let $g \in \mathbb{Z}[x]$ and for all polynomial $g_{i} \in g$, is convex and

$$
S_{B}=\left\{x \in \mathbb{Z}^{n} \mid g_{i}(x) \leq 0, i \in M, l \leq x \leq u\right\}
$$

then

$$
S_{B}=\left(P_{[g]} \cap\{(x, y) \mid y \leq 0\}\right) \cap \mathbb{Z}^{n}
$$

\section{The Existence of Optimal Value in Combinatorial Polynomial}

After obtained the set of feasible (polyhedra) of the problem (2), the next will be discussed the set of all minimum values of the set of integer points that make $f$ minimum. Namely determine the condition that there is $x^{0} \in S_{B}$ is the completion of equation (2) and whether the condition so that there is an integer $f\left(x^{0}\right)$ such that $f\left(x^{0}\right) \leq f(x)$ for all $x \in S_{B}$ with

$$
S_{B}=\left\{x \in \mathbb{Z}^{n} \mid g_{i}(x) \leq 0, i \in M, l \leq x \leq u\right\}
$$

Consider function $f: S_{B} \rightarrow \mathbb{Z}$, will be shown there is a $x^{0} \in S_{B}$ so $f\left(x^{0}\right) \in \mathbb{Z}$ and $f\left(x^{0}\right) \leq f(x)$ for all $x \in S_{B}$.

$$
\left(x^{0}, f\left(x^{0}\right)\right) \in\left\{(x, f(x)) \mid g_{i}(x) \leq 0, i \in M, l \leq x \leq u\right\}
$$

and $f\left(x^{0}\right)$ is the optimum value, not necessarily the global optimal value of $f(x)$. To examine the set of all integers and minimum points guarantee of polynomial combinatorial optimization problem (2) has the optimum value is as follows:

1. By adopting theorem on the existence of non-linear program over the real, which states that every $f$ continuous on the set of the compact will achieve that, $f: S_{B} \rightarrow \mathbb{Z}, f$ has at least one optimum in $S$ if $S$ compact namely (closed and limited) and $f$ is continuous.

$$
S_{B}=\left\{x \in \mathbb{Z}^{n} \mid g_{i}(x) \leq 0, i \in M, l \leq x \leq u\right\}
$$

not empty.

2. If $u \geq 0$ and $l \geq 0$ and $l, u \in \mathbb{Z}^{n}, l+1>0$ in box $l \leq x \leq u$ then there is $g(x) \leq 0$ because $x \in \mathbb{Z}^{n} \cap[1, u]$ can be transfer to non-negative variable $l^{\prime}=x-1$ with upper bound $u^{\prime}=u-1$.

3. $f$ polynomial then $f$ continuous on $l \leq x \leq u$ then there is $x^{*} \in[l, u]$ such that $f\left(x^{*}\right) \leq f(x)$ for all $x \in\left[l^{\prime}, u^{\prime}\right], x \in[1, u]$ (for $f$ minimum), $f\left(x^{*}\right) \geq f(x)$ (for $f$ maximum).

\section{REFERENCES}

[1] N. Alon and M. Tarsi, "Colorings and orientations of graph," Combinatorica, vol. 12, no. 2, pp. 125-134, 1992.

[2] N. Alon, "Combinatorial nullstellensatz," Combinatorics, Probability and Computing, vol. 8, pp. 7-29, 1999. 
[3] J. A. D. Loera, P. N. Malkin, and P. A. Parrilo, "Computation with polynomial equations and inequalities arising in combinatorial optimization," The IMA Volumes in Mathematics and its Applications, pp. 447-481, 2012. 